\title{
La posesión externa en Mazahua ${ }^{1}$
}

\author{
The external possession in Mazahua
}

Armando Mora-Bustos ${ }^{2}$

\begin{abstract}
Resumen
Las construcciones de posesión externa en mazahua se codifican a través de un sufijo de dativo; estas construcciones se comportan sintácticamente como las oraciones de objeto indirecto. Los rasgos bajo los cuales se presenta la construcción de posesión externa son: la frase nominal que expresa la relación poseído-poseedor lleva una marca de posesión y esta frase aparece básicamente en posición postverbal. La entidad poseída es semánticamente muy amplia, esto es, relaciones de parte todo, relaciones de parentesco y posesiones en general, como comida, ropa, dinero, animales, vivienda, instrumentos laborales, musicales y de cultivos. El poseedor es una entidad animada y puede aparecer expresado en primera, segunda y tercera persona. Los estados de cosas expresados en las construcciones codifican actividades, achievements, acomplishments que en lo regular pueden o no afectar al paciente. La posesión externa no se codifica con locativos y con verbos de estado. El evento no debe ser reflexivo y no debe haber correferencia entre el sujeto y el poseedor.
\end{abstract}

Palabras clave: Mazahua. Dativo. Posesión externa. Objeto directo. Objeto indirecto.

\begin{abstract}
The constructions of external possession in mazahua are coded as dative suffix; these structures behave syntactically as indirect object. The features under which presents the construction of external possession are: the nominal phrase that expresses the relationship possessed-possessor bears a mark of possession and basically this sentence appear in postverbal position. The possessed entity is semantically very wide, i.e. relations part all, kinship relationships and possessions in general, such as food, clothing, money, animals, housing, labor, musical instruments and crops. The possessor is an animate entity and may appear in first, second and third person. The states of things expressed in constructions code activities, achievements, accomplishments that usually can or cannot affect the patient. External possession is not coded with locative complements and state verbs. The event should not be reflexive and the subject is not cross-referenced with possessed NP.
\end{abstract}

Keywords: Mazahua. Dative. External possession. Direct object. Indirect object.

\footnotetext{
${ }^{1}$ Quiero agradecer la colaboración y la disposición en el proceso de elicitación de los datos presentados en este trabajo a Natalia Nicolasa Flores Crescenciano y a Marcelina Gregoria Romero Rodríguez, hablantes nativas del mazahua de San Pedro Potla. Algunas ideas de este trabajo fueron presentada en el Seminario de Complejidad Sintáctica 2014. Universidad de Sonora.

${ }^{2}$ Universidad Autónoma Metropolitana. Unidad Iztapalapa (México).
} 


\section{Introducción}

El mazahua es una lengua Otomangue del subgrupo Otopame. La variante aquí estudiada pertenece a San Pedro Potla, municipio de Temascalcingo, Estado de México. Ésta es una lengua nominativo-acusativa; igualmente es una lengua activo-estativa, ya que presenta un alineamiento escindido en las construcciones intransitivas con verbos estativos y pacientivos. Las relaciones gramaticales se codifican en el núcleo verbal a través de un complejo conjunto de prefijos y de sufijos respectivamente. El prefijo portmanteau junto al sujeto expresa tiempo, aspecto y modo; por otra parte, la unidad léxica verbal no dispone de un espacio morfológico para codificar a la vez el objeto directo y el objeto indirecto, de tal modo que sólo se exhibe uno de los dos. En las frases nominales posesivas el poseedor aparece expresado en un prefijo que se ancla al nominal que denota la entidad poseída. Esta lengua exhibe un orden de constituyentes VOS en las construcciones transitivas, VO-OI-S en las construcciones ditransitivas y VS en las intransitivas. En los prefijos de tiempo, modo, aspecto y persona, y en los de posesión se configuran los tonos de nivel, alto o bajo, mientras que los tonos de contorno, ascendente y descendente, aparecen en las unidades gramaticales léxicas.

El objetivo de este este trabajo es describir y caracterizar las construcciones de posesión externa en mazahua. Payne y Barshi (1999) describen la posesión externa como aquellas construcciones en que la relación semántica poseedorposeído se expresa codificando al poseedor como un argumento central del verbo y en un constituyente separado del que codifica al poseído. Dentro de las lenguas originarias de México, este recurso morfosintáctico, está registrado a través de cuatro mecanismos gramaticales (Palancar y Zavala 2013). En el zoque de Tectapán, como en (1a), el poseedor se codifica como argumento central y el poseído como oblicuo; en otomí de San Ildelfonso Tultepec, como en (1b), el poseedor se codifica como objeto indirecto, por intermedio de una forma de dativo; en Soteapaneco, como en (1c), el sustantivo que expresa lo poseído se incorpora (Mithun, 1984), en el verbo dejando vacante una posición argumental, la cual es ocupada por el poseedor sin provocar ningún cambio en la valencia verbal, esto es, de acuerdo con Palancar y Zavala 2013, se presenta un reacomodo en la estructura argumental sin alterar la valencia original. En náhuatl de Amanalco, como en (1d), el poseedor de la frase nominal pasa, a través de un aplicativo, a ocupar la posición de objeto primario.

1 (a). ngyoka?u mij yõmopit

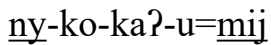
n-yomo? $=$ pit
2ABS-APL:MAL-morir-COM=PRO2ABS
2PSR-mujer $=\mathrm{con}^{3}$

\footnotetext{
${ }^{3}$ Abreviaturas: ART artículo; DAT dativo; DEL delimitativo; DEM demostrativo; DIM diminutivo; DL dual; E enfático; EXCL exclusivo; FOC foco ; FUT futuro; IND indefinido; LOC locativo;
} 
'Se te murió tu mujer.' (Lit. 'Te moriste con/por tu mujer.')

(Palancar y Zavala, 2013)

(b). bi=ñü's-k-a=má ts'ói

3.PSD $=$ llenar.TA-1DAT-D $=1$ POS olla

'Me llenó mi olla.' (Palancar y Zavala 2013)

(c). mi=puy-ku.wi?ks-w

$2 \mathrm{ABS}=$ pie-doblarse-COM

'Te torciste el pie.' (Boudreault 2009 en Palancar y Zavala, 2013)

(d). ti-ne:ch-ihti-li

2SG-OP1SG-ver-APL.PRF POS1sG-niño-SG.PSDO

'Me cuidaste a mi hijo.' (Lit. 'Me viste a mi hijo.') (Peralta, 2013)

El mazahua de San Pedro Potla, de manera similar al otomí de San Ildelfonso Tultepec (Palancar 2008 y 2009), exhibe el segundo tipo para codificar la posesión externa, esto es, esta lengua emplea la estrategia con dativo, el poseedor aparece codificado argumentalmente en el verbo con un sufijo de dativo, como en (2b). La relación que se establece entre el poseedor y el poseído es de interdependencia, tanto en (2a) como en (2b). Formalmente, se establece una conexión entre el poseedor y el poseído en (2a), a través de una forma de posesión interna; mientras que en $(2 b)$ se hace con una forma de posesión externa.

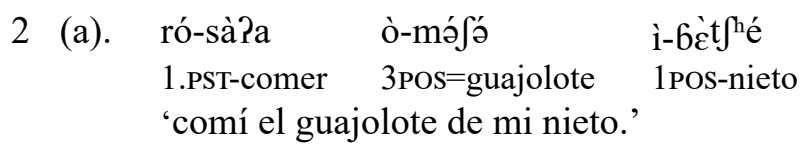
(b). ró-sà-p'ó
ò-mò̀’’
ì-bìthé
1.PST-comer-3DAT 3POS-guajolote
'le comí su guajolote a mi nieto.'
1POS-nieto

Este artículo está organizado de la siguiente manera. Primero la estructura de la frase nominal posesiva, el paradigma de sufijos de objeto y de dativo. Luego las marcas de posesión interna, la codificación de la posesión externa, el dativo y la posesión externa. Por último, la distribución de la entidad poseída, las propiedades de la posesión externa y las conclusiones.

DIM diminutivo; NEG negación; NP nombre propio; NUM numeral; OBJ objeto; Op objeto primário; PL plural; POS posesivo; PRED predicativo; PRON pronombre; PRS presente; PST pasado; PTL puntual; REL relativo; REFLEX reflexivo ; S relación s; SUB sujeto; SG singular; тот totalizador. 


\section{Estructura de la frase nominal posesiva}

La frase nominal está formada por un nominal nuclear que denota a la entidad poseída y un nominal en función modificadora que expresa a la entidad poseedora. La relación entre poseído y poseedor se establece por yuxtaposición, prefijación o por tono. Semánticamente, entre una construcción de posesión interna y de posesión externa se genera un efecto de énfasis de la afectación (Payne y Barshi 1999; Aikhenvald y Dixon 2013); el participante marcado con el dativo se encuentra en una situación de afectación de carácter benefactiva o malefactiva, como en (3). En (3a), dentro de la frase nominal posesiva que aparece en función de objeto directo, núwã̀mã á íùt'íi 'su milpa de mi niña', se expresa una relación de posesión entre el poseedor y lo poseído a través de una forma de posesión interna. Por su parte, en (3b), se genera una forma de posesión externa. El poseedor, iff' $i i$ 'mi niño' aparece codificado en el verbo a través de la forma de dativo $-p$ ' $\dot{t}$ y en el prefijo ó- del nominal que expresa la entidad poseída. El poseedor marcado con el dativo enfatiza la relación que existe entre poseedor y poseído y, a su vez, el poseedor está directamente afectado por el sentido del verbo cuidar.
1PRON 1PRS-cuidar=1SUJ.E 'cuido la milpa de mi niña.'

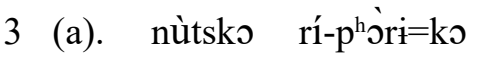
nú=wãmã $\quad$ í-fùt' 'ii $=\mathrm{k}$ o
3POS=milpa 1 POS-niña=POS.E
(b). nùtskó rí-p $\mathrm{p}^{\mathrm{h}} \mathrm{j}-\mathrm{p}$ ' $\mathrm{i}=\mathrm{i}$ o
'le cuido los toros a mi niño.'
1PRON 1.PRS-cuidar-3DAT $=1$ SUJ.E
ó-ndzîni
í-t’’’ii
3Pos-toro 1 1Pos-niño

La construcción codificada en forma de posesión interna, como en (4a) es ambigua, puede denotar más de un sentido. El hablante pudo haber comprado los pollos para regalarlos a otra persona, el hablante puede o no tener conocimiento de quién es esa segunda persona, simplemente puede haber una etiqueta que dice 'lleva tus pollos'. Por su parte, en (4b), el poseedor, la segunda persona, está completamente afectado por la acción de comprar; en este caso, efectivamente, el hablante sabe de quién se trata esta segunda persona (Fried 1999; Palancar 2009).
4 (a). rá-t’òmi ì̀-ndzôñí
1FUT-comprar 2POs-pollo
'compraré tus pollos.'
(b). ij'á=ró-t'òn-g ${ }^{w i} \quad$ ì-ndzว̀n๊
NEG $=1$ PST-comprar-2DAT 2POS-flor
'no te compré tus flores.'


La posición natural de la frase nominal que expresa tanto a la entidad poseída como al poseedor se ubica en posición postverbal, como en (5a) y (5c); no obstante, esta frase nominal posesiva se puede ubicar en posición preverbal. En este último caso, generalmente, un artículo o un demostrativo anteceden al prefijo que exhibe al poseedor, como en (5b) y (5d).

5
(a). nùtsḱ́
1.PRON
ró-pìr-p'o
'le golpee el pecho al señor.'
o-tìmí
nù-bězó
3Pos-pecho
ARTSG=Señor

(b). nù=ó-tìmí

nù=6と̌zó

ij' $\mathrm{a}=$ ró-pìr-p'o=k $\mathrm{o}=\mathrm{nu}$

ARTSG $=3$ POS-pecho $\quad$ ARTSG $=$ señor

NEG $=1 . P S T$-golpear-3DAT $=1$ suB.E $=$ DEM

'no le golpee el pecho al señor.'

(c). rá-t'tr $\mathrm{ss}^{\prime} \mathrm{i}=\mathrm{k}$

1-FUT-colgar=1sUB.E

jò=6ítú

jò=t’’̀i

'colgaré la ropa del niño.'
(d). nùjó=6ítú
jò=t'ri
rá-th̃ $\tilde{\mathrm{ts}}^{\prime} \mathrm{i}^{\mathrm{i}}=\mathrm{kj}=\mathrm{jo}$
ARTSG.3POS=ropa
ARTPL=niño
1.FUT-colgar $=1$ SUB.E $=$ DEM
'le colgaré la ropa al niño.'

Las dos entidades semánticas que aparecen dentro de la frase nominal posesiva expresan varias clases de relaciones semánticas y están codificadas a partir de varios patrones (Arellanes, Ortiz, Mora y García 2015), esto es, yuxtaposición, nùj 'ówi nùMária 'la hermana de María', como en (6a); expresión morfológica, un prefijo en el núcleo, ómério nùLupita 'el dinero de Lupita', como en (6b); y a través de un tono alto, núñáã à nùMária 'la voz de María', como en (6c).

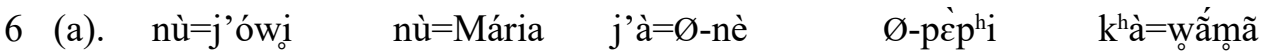 3POS=hermana $\quad \mathrm{ARTSG}=$ Maria $\quad \mathrm{NEG}=3$. PRS-querer 3.PRS.trabajar $\quad$ LOC=milpa 'la hermana de María no quiere trabajar en la milpa.'

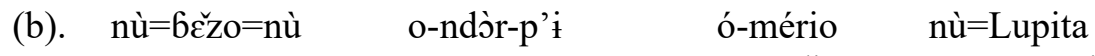 ARTSG=señor=DEM 3.PST-agarrar-3DAT 3POS-dinero ARTSG=Lupita 'el señor le agarró el dinero a Lupita.'
(c). nú=ñá?ã nù=Mária rí-Pòrə=hi $\quad \mathrm{k}$ à= $=\varnothing=$ ndzúmi $=$ hi 3.POS $=\mathrm{vOZ} \quad$ ARTSG $=$ Maria $\quad 1 . \mathrm{PRS}-\mathrm{O}$ ir $=\mathrm{PL} \quad \mathrm{LOC}=1 . \mathrm{POS}=\mathrm{casa}=\mathrm{PL}$ 'la voz de María la escuchamos en nuestra casa.'




\section{Sufijos de objeto y de dativo}

En mazahua existe un amplio repertorio de sufijos verbales de objeto y de dativo, como en (7) (Stewart 1966; Knapp 2008; Fidencio 2012 y Mora 2012); este conjunto de alomorfos están condicionados por procesos morfofonológicos. Para mayor detalle en este punto se puede ver Knapp (2008).

DATIVO

1

/-gV/ -kV, -k $\mathrm{k}^{\mathrm{h}} \mathrm{V},-\mathrm{zV},-\mathrm{tsV}$

/-kV/ -ngV, -zV, -tsV

$2 \quad /-k^{\prime} \mathrm{V} / \mathrm{-ts}{ }^{\prime} \mathrm{V}$

/-?k’V/ -ts'V, -k’V

$3-\varnothing$

/-pV/ -mbV, - p’V, - p $^{\mathrm{h}} \mathrm{V}$

A partir de la naturaleza transitiva del verbo, como en (8a) y (8b), o ditransitiva, como en (8c) y (8d), se exhibe en la base verbal el sufijo que codifica al objeto o al dativo. La tercera persona de objeto se marca con un morfema cero, como en (8a); en (8b) aparece el sufijo que expresa al objeto de primera persona. En los verbos pǒní 'robar' de (8c) y péñe 'enviar' de (8d) se sufijan los dativos correspondientes a la primera y segunda persona respectivamente.
8 (a). ò- $\int \hat{\varepsilon} \mathrm{t} \int \grave{\mathrm{i}}-\varnothing \quad$ nù=Mária
3.PST-pellizcar-3OBJ ARTSG=María
'pellizcó a María.'

(b). ò- $-\hat{\varepsilon}$-tsì

3.PST-pellizcar-1OBJ

'me pellizcó.'
(c). ó-ndà-pò-ngi=hi jò=ngóní nùjò ná-t’’’?f́t
2.PST-TOT-robar-1DAT $=$ PL $\quad$ ARTPL=pollo REL PRS.PRED-blanco 'me robaron todos los pollos blancos'
(d). ró-pền-k'e rí-mà $\quad$ Ø-t 5 'òmi $\quad$ jò=múru 1.PST-enviar-2DAT 2.FUT-ir 2.FUT-comprar $\quad$ ARTPL $=$ huevo 'te mandé a comprar huevos'

Morfológicamente, en el complejo verbal sólo hay un espacio o hueco estructural para sufijar una relación gramatical; de tal modo que en una construcción ditransitiva se codifica únicamente al dativo, como en (9a) y (9b). En caso de que el recipiente se encuentre expresado en una frase nominal plena, como en (9a), el dativo hace referencia cruzada con esta frase nominal. 


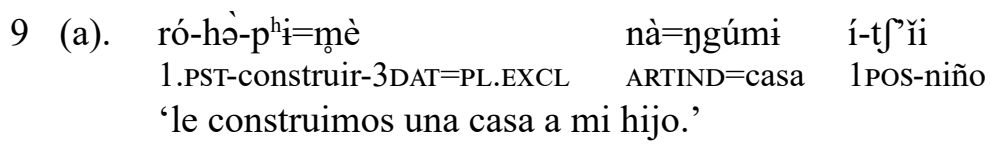

(b). ó-ngìs-p'í=hi

3.PST-quitar-3DAT $=$ PL

'se lo quitaron.'

\section{Marcas de posesión}

En mazahua de San Pedro Potla hay un gran repertorio de formas posesivas, como en (10), que se prefijan al sustantivo nuclear de la frase nominal. Básicamente, el rasgo que identifica a las diferentes personas es suprasegmental, esto es, un tono alto o un tono bajo.

10 Prefijos de posesión

1 í- ní- mí-

2 ì- nì- mì-

3 ò- nú- jó-

En los ejemplos de (11) se ilustra, con algunos nominales o entidades poseídas, la posición prefijal de estas unidades morfológicas que codifican al poseedor. En contraste con otras variantes del mazahua (Quintana 2012 y Vargas 2013), en San Pedro Potla no se ha encontrado ningún tipo de asociación o relación semántica entre estos prefijos y la unidad léxica con la que aparecen.
11 (a). $\quad \begin{aligned} & \text { nú-phàdł́ } \\ & \text { 3Pos-caballo } \\ & \text { 'su caballo.' }\end{aligned}$
(c). í-bítu
1POS-ropa
'mi ropa.'

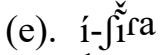 1POS-esposo 'mi esposo.'
(b). jó-Pànə
3Pos-guajolote
'sus guajolotes.'
(d). nì-tshêze
2pos-falda
'tu falda.'
(f). mì-pále 1Pos-abuelo 'mi abuelo.'

Dentro de las frases nominales, la marca de posesión es la vía que explicita la posesión interna. La frase nominal que expresa al poseedor y a la entidad poseída aparecen antes del verbo, como en (12b) y (12d), o después del verbo, como en (12a) y (12c). En todos estos casos se establece una relación de posesión entre la entidad poseída y el poseedor. Dentro del dominio de las entidades que pueden ser poseídas, entran, sin restricción semántica, todos los objetos susceptibles de ser poseídos por un participante que tiene la capacidad 
de apropiárselos. Las relaciones semánticas establecidas en (12a) relación de parentesco, $(12 \mathrm{~b})$ relación de concepto de propiedad inalienable, (12c) relación de partes del cuerpo y (12d) relación de concepto de propiedad alienable (Arellanes, Ortiz, Mora y García 2015) están expresadas en una frase nominal cuya función gramatical es la de objeto directo.

$\begin{array}{llll}12 \text { (a). } & \text { nù=Bèrná } & \text { jâ= }=\text {-Pə̀t'á } & \text { nú-t'ǐi } \\ \text { ARTSG=Berna } & \text { PTL=3.PRS-curar } & \text { 3POS-niño }\end{array}$

'Berna cura a su hijo.'

(b). nú-j’óo nù=Rósa ó-sàbí

3POS-perro ARTSG-Rosa 3.PST-morder

'el perro de la señora Rosa lo mordió.'

(c). rí-pìt'i í-tfîmi

1.PRS-golpear 1POS-pecho

'golpeo mi pecho.'

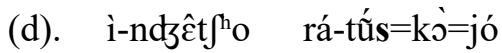

2Pos-maíz 1.FUT-llevar=1SUJ.E= DEM

'llevaré tu maíz.'

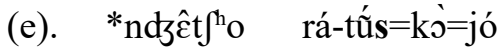

maíz 1.FUT-llevar=1suJ.E=DEM

'llevaré tu maíz.

En las construcciones de posesión interna, como en (12), y de posesión externa, como en (13), el prefijo de posesión debe aparecer de manera obligatoria en la entidad poseída. Las oraciones que no presenten esta unidad afijal, como en (12e) y (13e), son anómalas, en cuanto no expresan el sentido de posesión. Sin la marca de posesión, el sentido de oración (12d) es 'llevaré maíz' y el de (13d) 'el viento aventó el sombrero'. La tendencia, en los casos de posesión externa, como en (13), es que las frases nominales que expresan al poseedor y al poseído aparezcan después del verbo.

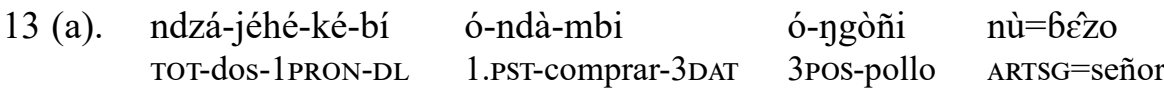
'él y yo le compramos los pollos al señor.'

(b). ró-k'àhki-Pts'í ì-6ítu

1.PST-echar agua-2DAT 2POS-ropa

'yo te mojé tu ropa.' 

(c). nù=ndằmã ó-pà-ngí í-th'
ARTSG=viento 3.PST-aventar-1DAT 1 POS-sombrero
'el viento me aventó mi sombrero.'
(d). í-th fhù̀ $^{\prime} \dot{i} \quad$ nù=ndằmã $\quad$ ó-pà-ngí 1POS-sombrero ARTSG=viento 3.PST-aventar-1DAT 'el viento me aventó mi sombrero.'
(e). *f'û̀s'i nù=ndằmã ó-pà-ngí sombrero ARTSG=viento 3.PST-aventar-1DAT 'el viento me aventó mi sombrero.'

Cómo ya fue mencionado más arriba, sintácticamente la forma de la posesión interna, como en (12), está expresada en una frase nominal que funciona como objeto directo, mientras que en la forma de posesión externa, como en (13), la entidad poseída está expresada en una frase nominal que funciona como objeto directo y el poseedor, codificado en el afijo de dativo, se comporta como un típico objeto indirecto. Este dativo hace referencia cruzada con la frase nominal plena que expresa al recipiente. En (13a), ndzájéhékébi óndàmbi óngòñi nù6êzo, 'él y yo compramos los pollos del señor', la frase nominal óngòñi 'sus pollos' funciona como objeto directo; el sufijo de dativo -mbi y la frase nominal nùbêzo 'el señor' hacen referencia cruzada y funcionan como objeto indirecto.

\section{Codificación de la posesión externa}

Morfológicamente la marca de posesión externa se codifica por medio de un sufijo de dativo que aparece en el núcleo verbal. El sufijo que codifica al dativo termina en vocal y el prefijo que exhibe al poseedor está codificado, igualmente, en una vocal; entre estas dos vocales que se encuentran en posición adyacente se genera un proceso de asimilación regresiva. En (14a) la vocal $i$ - del prefijo que expresa al poseedor es asimilada por la vocal del sufijo de dativo -zi. El mismo proceso de asimilación ocurre en (14b); la marca del poseedor de la entidad $o$-məfə 'guajolote' es asimilada por la vocal del sufijo de dativo - $p$ 'o.

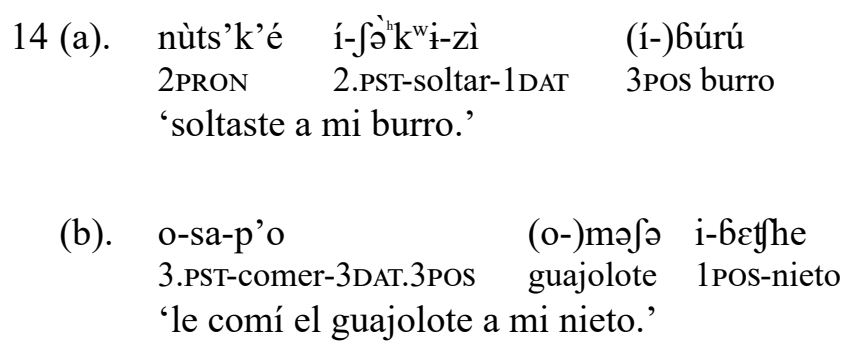


Generalmente, la primera consonante de la entidad poseída se palataliza por influencia del prefijo que codifica al poseedor; así la consonante dental oclusiva de t'ǐri 'niño', de (15a), y timi 'pecho' de (15b) se palatalizan: $t$ '’ '̌i $2 i$ y t' estos casos, generalmente, el prefijo de posesivo no aparece como en (15).

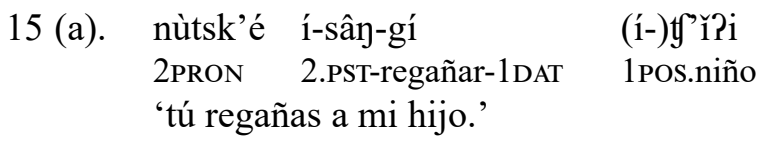
(b). o-t fin-ki (í-)t' imi
3.PST-golpear-1DAT 1POS.pecho
'me golpeó el pecho.'

Por lo general, tras la asimilación de la vocal que codifica la posesión, aparece un morfema de posesión enfática, como en (16a). El enclítico $=k^{h} \supset$ que aparece en la frase nominal bituk $^{h} \mathrm{o}$ ' $m i$ ropa' de (16a) enfatiza al poseedor; este enclítico de énfasis del poseedor también aparece en otros contextos sintácticos: en (16b) la frase posesiva ij'óôk'e 'tu perro' se encuentra en posición inicial de la oración y funciona como sujeto gramatical, el enclítico $=k$ 'e genera, también, un efecto de énfasis sobre el poseedor.
16 (a). nù= =úba
$\varnothing$-tfòr-ki
bítu= $\mathrm{k}^{\mathrm{h}} \mathrm{o}$
ARTSG=Juan 3.PRS-doblar-1DAT.1POS
ropa $=1$ POS.E
'Juan dobla mi ropa.'
(b). ì-j'ó?o=k'é
ndà-Ø-zà-kì
í-ndzôñi
2POS-perro $=2 \mathrm{POS}$.
TOT-3.PST-comer-1DAT
1POS-pollo
'tu perro se comió todos mis pollos.'

La tendencia para que aparezca el dativo que expresa la posesión externa, radica en que la frase nominal posesiva se encuentre en posición adyacente al verbo; sin embargo, el morfema de dativo puede coaparecer con un clítico delimitativo, $=t^{h} o$, como en (17a); uno de plural, $=h i$, como en (17b); uno de énfasis de dativo, $=t s i$, como en $(17 \mathrm{c})$, o uno de énfasis de sujeto, $=k$ ' $\supset$, como en (17d). Si entre el verbo y la frase posesiva se ubica un adjunto, $j$ ' $\hat{p} p a ̀ d f$ 'con una aguja', como en (17e) es improbable que aparezca el sufijo de dativo.
17 (a). ó-mbón-ki=tho
í-t'âp ${ }^{\text {hì }}$
3.PST-sobrar-1DAT=DEL
1Pos-pulque
'sobró mi pulque.' 

(b). ó-má Ø-ngór-ki=hi t’
3.PST-ir 3.PST-encerrar-1DAT=PL.1POS niño 'encerraron a mi hijo.'
(c). ó-mbor-ki=tsi í-ngôñ
3.PST-matar-1DAT=1DAT.E 1POS-pollo
'mató a mi pollo.'
(d). rá-má $\quad$-pè-mbe=k’o ò-bítu nù=t'íi
1.FUT-ir 1.FUT-lavar-3DAT=1sUB.E 3.POS-ropa $\quad A R T S G=$ niño 'le lavaré su ropa al niño.'

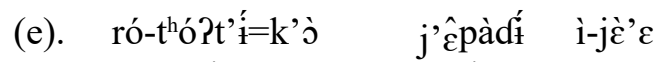
1.PST-picar=1sUB.E aguja 2.POS.mano
'piqué tu mano con una aguja.'

Dentro de las construcciones de posesión externa, la frase nominal que expresa la entidad poseída tiende a estar ubicada en posición posverbal, como en (18a). La codificación del sufijo de dativo - $p$ 'o está condicionado en parte porque la frase nominal posesiva ó $p^{h}$ âdi nùbčzo 'el caballo del señor' se ubica en posición postverbal. Esta frase nominal, en (18b), se encuentra ubicada en posición inicial, en consecuencia el sufijo de dativo no aparece codificado en el verbo; las construcciones de posesión externa no son posibles si la frase nominal posesiva se encuentra en posición inicial de la oración, como en (18c).

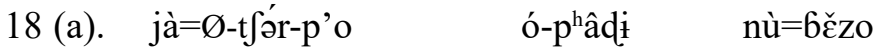 PLT=3.PRS-montar-3DAT 3POS-caballo $\quad$ ARTSG $=$ señor 'le monta el caballo del señor.'

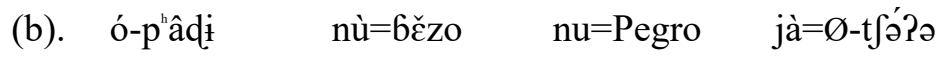
3POS-caballo ARTSG $=$ señor $\quad$ ARTSG $=$ Pedro $\quad$ PLT=3PRS-montar
'Pedro monta el caballo del señor.'

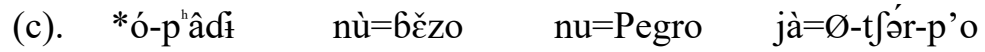 3POS-caballo $\quad$ ARTSG $=$ señor $\quad$ ARTSG $=$ Pedro $\quad$ PLT $=3$ PRS-montar-3DAT 'Pedro monta el caballo del señor.'

Los contextos gramaticales en los cuales no son posibles las construcciones de posesión externa están relacionados con los siguientes hechos. Si el sujeto gramatical es correferente con la entidad poseída, como en (19), no aparece la marca de la posesión externa. El sujeto gramatical de (19a) está expresado por 
la primera persona del singular y por la segunda persona del singular en (19b); en las dos oraciones estos sujetos, señalados con el subíndice, son correferentes con la entidad poseedora, es decir, el poseedor corresponde a la primera y la segunda persona del singular respectivamente. La codificación del sufijo del dativo en este tipo de construcciones con sujeto y poseedor correfentes genera oraciones agramaticales, como en $(19 \mathrm{c})$.

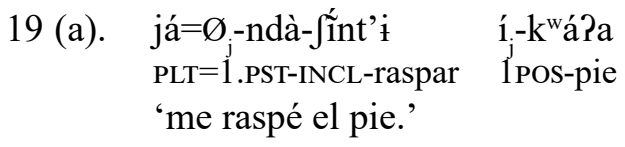

(b). ì -j'óki-kne $\quad i_{i}-\mathrm{j}$ ' $\hat{\varepsilon}$ ? $\varepsilon$

2.PST-cortar-2SUJ.E 2POS-dedo

'te cortaste tu dedo.'

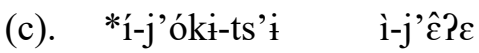

2.PST-cortar-2DAT 2POS-dedo

'te cortaste el dedo.'

En las construcciones reflexivas, como en (20a) y (20b), no aparece la marca de posesión externa. En estas oraciones aparece el enclítico $=t s^{\prime} \grave{\varepsilon}$ que codifica la propiedad de reflexividad por parte del sujeto gramatical. La razón para que no se codifique el clítico de dativo en (20), es por la misma naturaleza gramatical descrita en los ejemplos de (19); el sujeto gramatical y el poseedor son correferentes, de tal modo que no puede aparecer el sufijo de dativo, como en $(20 c)$.

20 (a). ró-hándi=ts' $\grave{\varepsilon} \quad$ míi k’à=ñéhe

1.PST-ver=REFLEX cara LOC=espejo

'me vi la cara en el espejo.'

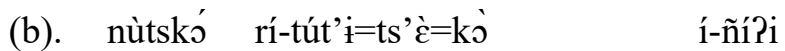

1PRON 1.PRS-peinar=REFLEX $=1$ SUJ.E 1 POS-cabeza

'me peino mi cabeza.'

(c). *rí-tún-ki=ts' $\grave{\varepsilon} \quad$ í-ñíi

1.PRS-peinar-1DAT=REFLEX 1POS-cabeza

'me peino mi cabeza.'

El poseedor que aparece dentro de un complemento locativo no se codifica dentro del complejo verbal como dativo de posesión externa, como en (21). Como parte del complemento locativo k'àìwâmãa 'en tu milpa' (21a), aparece una relación que se establece entre el poseedor y la entidad poseída, pero en este 
tipo de complemento no es posible que el poseedor se codifique en forma del dativo como en (21b).

\begin{tabular}{|c|c|c|c|c|}
\hline 21 (a). & $\begin{array}{l}\text { Pedro } \\
\text { Pedro }\end{array}$ & $\begin{array}{l}\text { já }=\varnothing-\text { má } \\
\text { PLT }=3 \text {.PRS-ir }\end{array}$ & $\begin{array}{l}\text { Ø-pêp } \mathrm{p}^{\mathrm{h}} \mathrm{i} \\
\text { 3.PRS-trabajar }\end{array}$ & $\begin{array}{l}\text { k'à=ì-wâ̂mãa } \\
\text { LOC=2POS-milpa }\end{array}$ \\
\hline
\end{tabular}
(b). $\quad{ }^{*} \varnothing-{ }^{\mathrm{h}} \mathrm{i}-\mathrm{p}^{\mathrm{h}} \mathbf{i}$
k’à=nú-ñíîi
3.PRS-abrazar-3DAT $\quad$ LOC=3POS-cabeza
'abraza su cabeza.'

\section{Dativo y posesión externa}

Tanto las construcciones de objeto indirecto (Mora-Bustos 2015) como las de posesión externa, comparten un conjunto de propiedades gramaticales dentro de la estructura del complejo verbal. En (22a) y (22b), los verbos ditransitivos, específicamente los verbos de transferencia péndo 'ofrecer' y t'òmi 'comprar' se comportan morfológicamente de manera similar a los verbos que codifican posesión externa $t^{\text {h' }}$ 'ri 'destruir' y pej'e 'lavar' de (22c) y (22d). En las oraciones en las que aparecen estas unidades verbales, aparece codificado un sufijo de dativo - mbi: óndàndう̇mbi en (22b) y ómbèmpo en (22d); una frase nominal independiente que expresa al objeto directo óngòni 'los pollos' en (22b) y bitu 'su ropa' en (22d), y en otra al objeto indirecto, nùbêzo 'el señor' en (22b) y nù t'íi 'el niño' en (22d). Tanto en las construcciones de verbos ditransitivos, como en las de posesión externa, el dativo hace referencia cruzada con la frase que expresa al receptor como en (22b), óndàndj̇mbi óngòñi nùbeżo y con la que expresa al poseedor, como en (22d), nùMáría ómbèmpo $o_{x}$ bítu nù t'ǐi $i_{x}$

\begin{tabular}{|c|c|c|c|}
\hline 22 (a). & $\begin{array}{l}\text { ó-péndo-ts'ł } \\
\text { 3.PST-ofrecer-2.DAT }\end{array}$ & $\begin{array}{l}\text { jò }=\text { mũù̃ } \\
\text { ARTPL }=\text { calabaza }\end{array}$ & $\begin{array}{l}\text { è-Pégro } \\
\text { NP-Pedro }\end{array}$ \\
\hline
\end{tabular}

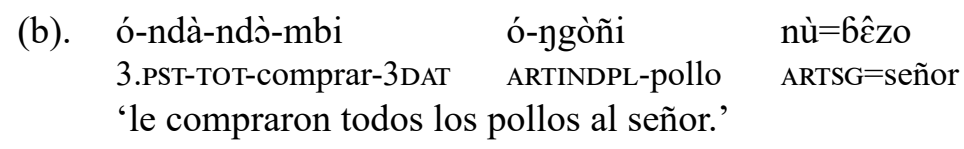
(c). ó-ndó-mpí ò-ggǔmí nù=Rósa
3.PST-destruír-3DAT $\quad 3$ POS-casa $\quad$ ARTSG=Rosa 'le destruyó su casa a Rosa.'
(d). nù=Máría ó-mbè-mpo bítu nù=t'ìi ARTSG $=$ María 3.PST-lavar-3DAT.3POS ropa $\quad \mathrm{ARTSG}=$ niño 'María le lavó su ropa al niño.'


El constituyente que funciona como objeto indirecto, nùt'it' 'ii 'el niño' de (23a) aparece después del objeto directo ót $t^{\grave{u}} u n s$ ' $\tilde{t}$ 'su sombrero'. El cambio de orden del objeto indirecto, en posición postverbal, como en (23b), no es posible. En una construcción de posesión externa, como en (24), ocurre lo mismo. En (24a), la entidad poseída está expresada en la frase nominal bitu 'ropa' que funciona como objeto directo; la entidad poseedora está expresada en la frase nominal nùfúba 'Juan' que funciona sintácticamente como un objeto indirecto, ya que no puede aparecer en una posición diferente que no sea la final, como en (24b).

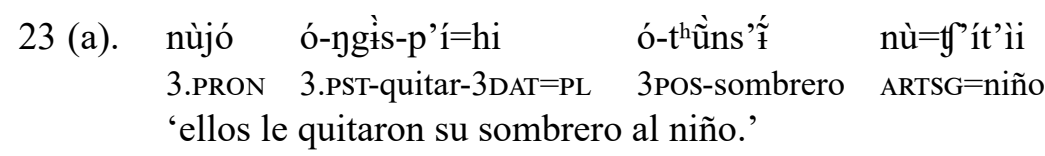

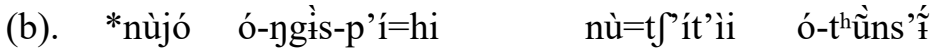
3.PRON 3.PST-quitar-3DAT $=$ PL ARTSG=niño 3POS-sombrero
'ellos le quitaron su sombrero al niño.'

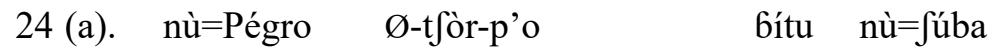 ARTSG $=$ Pedro 3.PST-doblar-3DAT.3POS ropa ARTSG $=J$ Juan $^{2}$ 'Pedro le dobla su ropa a Juan.'

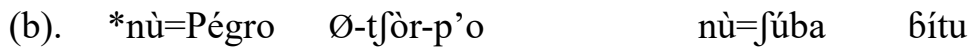 ARTSG $=$ Pedro 3.PST-doblar-3DAT.3POS ARTSG=Juan ropa 'Pedro le dobla la ropa a Juan.'

Tanto en construcciones de objeto indirecto, como en (25a), como en la de posesión externa, como en (25b), se omiten las frases nominales que expresan al recipiente y al poseedor; estos se recuperan en el contexto.

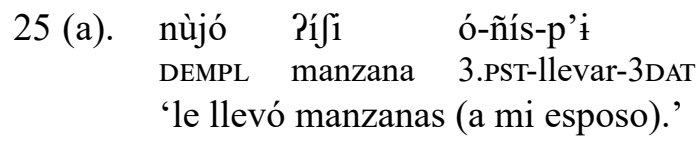

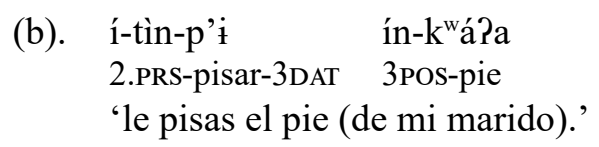

Esta lengua utiliza el recurso gramatical de las construcciones escindidas para focalizar o poner en relieve la información que se expresa en una forma gramatical. En (26a), a través del focalizador $\eta g e$, se pone en foco la información expresada en una forma de posesión interna, núifiskomi 'mi papel'. Igualmente, 
las construcciones de dativo y de posesión externa se focalizan utilizando este recurso gramatical. En (26b) se focaliza el recipiente, nùtsk'éhi 'ustedes', y en (26c), el poseedor $=k$ ' $\dot{t}$ 'eso'.

$\begin{array}{lllll}26 \text { (a). } & \text { ijó, } & \text { mbè nùk'í } & \text { rí-hódi=go } & \text { ygé=nú=1́-Jískomi } \\ \text { NEG pero REL } & 1 . \text { PRs-buscar-1sub.E } & \text { FOC=ARTSG=1 POS-papel } \\ \text { 'no, pero lo que yo busco es mi papel.' } & \end{array}$

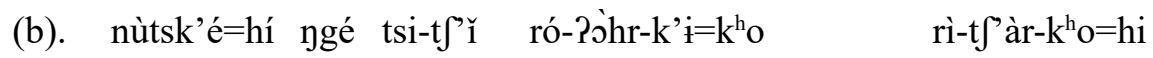
2.PRON=PL FOC DIM-hijo 1.PST-pedir-2.DAT=1.sUB.E 2.FUT-dar-1.DAT=PL hándzi měrio algo dinero 'a ustedes que son mis hijos les pedí que me dieran algo de dinero.'
(c). ja=nuj'a=k'i ja=nge=k'i ri-ts' ${ }^{\mathrm{h} a} a-p^{\prime} \dot{i}$ $\mathrm{nu}=$ ñoni $=\mathrm{j}{ }^{\prime} \mathrm{a}=\mathrm{k}$ 'i PTL $=$ hoy $=$ DEM $\quad$ PTL $=$ FOC $=$ REL $\quad$ 2.FUT-hacer-3DAT $3 \mathrm{POS}=$ comida $=\mathrm{PTL}=\mathrm{DEM}$ 'ahora, eso es lo que harás de comida.'

\section{Propiedades de la posesión externa}

Palancar (2008 y 2009) señala que para el otomí de San Ildefonso Tultepec la construcción de posesión externa es obligatoria, entre otras propiedades, cuando lo poseído es una parte del cuerpo o vestimenta propia; en mazahua las construcciones de posesión externa aparecen en contextos pragmáticos específicos, básicamente cuando el hablante quiere hacer énfasis en el poseedor y la frase nominal posesiva está compuesta por dos entidades (poseedor y poseído) semánticamente muy integrada (Aikhenvald y Dixon 2013); en consecuencia el espectro semántico de la entidad poseída es muy amplio, es decir, se encuentran relaciones de parte todo (parte del cuerpo) en (27a); relaciones de parentesco en (27b); y relaciones de alienabilidad e inalienabilidad en general: comida en (27c); ropa, dinero en (27d); animales en (27e); vivienda en (27f); partes de la vivienda en (27g) y (27h); instrumentos de labor doméstica en (27i); instrumentos musicales en (27j); e instrumentos de trabajo en (27k) y cultivos en (271).

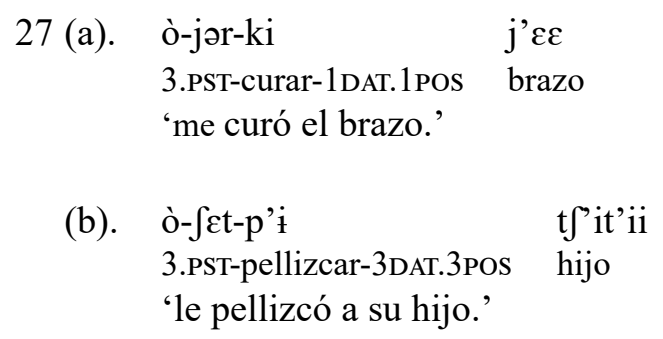




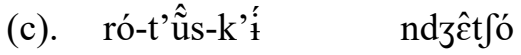

1.PST-llevar-2DAT 2POS.maíz

'te llevo tu maíz.'

(d). nù=Máría ó-mbè-mbo

bítu nù=t'ìi

ARTSG=Maria 3.PST-lavar-3DAT.3POs ropa $\quad \mathrm{ARTSG}=$ niño

'María le lavó la ropa al niño.'

(e). nù=6 $\hat{\varepsilon} z o=$ nù ò-ndòr-p'i ó -mério nù=Lupita

ARTSG=Señor=DEM 3.PST-agarrar-3DAT $\quad$ 3POS-dinero $\quad$ ARTSG $=$ Lupita

'el señor le agarró el dinero a Lupita.'

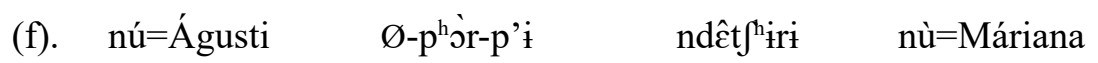
ARTSG $=$ Agustin 3. PRS-cuidar-3DAT 3POs.borrego ARTSG=Mariana

'Agustín le cuida los borregos a María.'

(g). í-t’'ỗn-kí=hi índzímí

2.PST-tumbar-1DAT=PL 1POS-casa

'me tumbaron mi casa.'

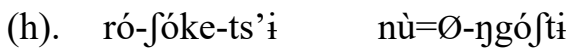

1.PST-abrir.2DAT ARTSG=2POS-puerta

'te abrí tu puerta.'

(i). ró-tũs-p'i k'ini

1.PST-cargar-3DAT.3Pos metate

'yo le cargo el metate a mi esposo'

(j). ì- $\mathrm{p}^{\mathrm{h}} \hat{\mathrm{c}} \mathrm{n}-\mathrm{k}^{\mathrm{w}} \dot{\mathrm{i}} \quad$ í-6ízi

2.PRS-tocar-1DAT 1 1 POS-guitarra

'tú me tocas mi guitarra.'

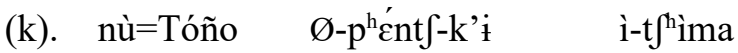

ARTSG=toño 3.PRS-agarrar-2DAT 2POS-lazo

'Antonio te agarra tu lazo.'

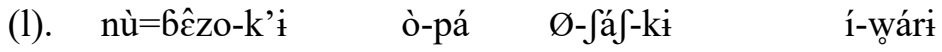

ARTSG=señor-DEM 3.PST-ir 3.PST-raspar-1DAT 1POS-maguey

'el señor me fue a raspar mis magueyes.' 
Aquí se ha mencionado que en las construcciones de posesión externa, la frase nominal que expresa al poseedor tiene restricciones de movimiento, como en (23) y (24); igualmente, se ha descrito, de (14) a (17), la forma de codificación de los prefijos de posesión. Con respecto a la posición de la frase nominal posesiva que exhibe a la entidad poseída y que funciona como objeto directo, se ha dicho que tiende aparecer en posición postverbal, óngôñ̃ 'sus pollos', como en (28a); sin embargo, en (28b), la frase posesiva en forma de posesión interna, núñá̃ ã nùMária 'su voz de María' y que funciona como objeto directo puede aparecer en posición preverbal; en esta posición también aparece la frase nominal que expresa al poseedor de una forma de posesión externa, núj 'é? $\varepsilon^{\prime}$ 'su mano', como en (28c). Entre (28d) y (28e) se hace un contraste de la posición del objeto directo en una construcción de posesión externa; la frase nominal posesiva, ikwá?a 'tu pie', que expresa la entidad poseída puede o no aparecer en posición adyacente al verbo.

\begin{tabular}{|c|c|c|c|c|}
\hline (a). & $\begin{array}{l}\text { nùstsk'ó } \\
\text { 1PRON }\end{array}$ & $\begin{array}{l}\text { ró-t'ว̀?-p'ò } \\
\text { 1.PST-encontrar-3DAT }\end{array}$ & $\begin{array}{l}\text { ó-ygôñ̃ } \\
\text { 3POS-pollo }\end{array}$ & $\begin{array}{l}\text { ì-súu } \\
\text { 2Pos-esposa }\end{array}$ \\
\hline
\end{tabular}

(b). nú-ñắ2ã nù=Mária rí-Pว̀rə=hi $\quad$ k’à= $=\varnothing-n d z u ́ m i=h i$ 3POS-voz ART=Maria 1. PRS-oir $=\mathrm{PL} \quad$ LOC $=1$. POS $=$ casa $=\mathrm{PL}$ 'su voz de María la escuchamos en nuestra casa'

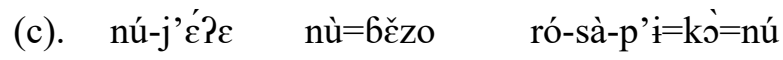
3POS-mano ARTSG=señor $\quad$ 1PST-morder-3DAT=1SUJ.E=DEMSG 'le mordí la mano al señor.'

(d). rá-Pə̀r-k’i ì-kwá?a

1.FUT-curar-2DAT 2POS-pie

'te curaré el pie.'

(e). i-kwa?a-k'e ra-Por-k'i

2POS-pie $=2$ POS.E $\quad$ 1.FUT-curar-2DAT

'te curaré el pie.'

Las construcciones de posesión externa aparecen con verbos que expresan diferentes estados de cosas, tales como actividades (29a), achievements (29b) y accomplishments (29c). Las construcciones de posesión externa no se generan con verbos de estado (29d).

29 (a). ò-j’ókí-zi ñìi

3.PST-cortar-1DAT cabeza

'el me cortó el cabello' 


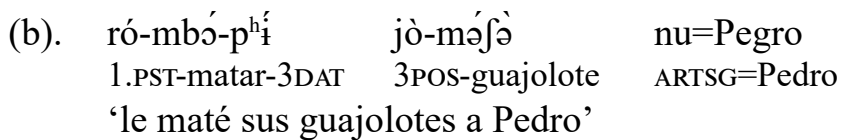

(c). ó-jər-ki j’'êc

3.PST-curar-1DAT.1POS brazo

'me curó el brazo'

(d). í-pènt $\int^{\prime 1} \quad$ í-pắnã̃ $=\beta \mathrm{i}$

2.PRS-tener agarrado 1 POS-camisa $=\mathrm{DL}$

'me tienes agarrada mi camisa'

En las construcciones de posesión externa, al encontrar como verbos nucleares unidades léxicas que están dotadas de un conjunto de rasgos semánticos inherentemente diversos, el objeto no está completamente afectado (Hopper y Thompson 1980; Dowty 1991; Beavers 2011,) en todos los casos de (29). Con los verbos de achievement, como pót’i 'matar' de (29b) y accomplishment, como at’a 'curar' de (29c), el grado de afectación del paciente es mayor que con los verbos de actividad, como óki 'cortar' de (29a).

A partir de la escala de jerarquía de rasgos que propone Haspelmath (1999), básicamente para un grupo de lenguas indoeuropeas, las construcciones de posesión externa del mazahua se distribuyen de la siguiente manera: (a). Animacidad del poseedor: éste se codifica en un pronombre de primera, segunda y tercera; y en un nombre propio. Todas las entidades poseedoras son animadas. (b). Situación: el paciente puede o no estar afectado. (c). Inalienabilidad de lo poseído: las entidades poseídas pueden ser las partes del cuerpo, la vestimenta y un gran conjunto de entidades que se encuentran al alcance del poseedor. (d). Relación gramatical de lo poseído: la entidad poseída únicamente se expresa en el objeto directo. Si se considera como punto de referencia a esta propuesta, translingüísticamente las lenguas mazahua y otomí (Palancar 2008) comparten, con respecto a las construcciones de posesión externa, prácticamente todos los rasgos de esta jerarquía. Una pequeña diferencia se centraría en el hecho de que en el mazahua el grupo semántico de las entidades poseídas es más amplio que el del otomí.

\section{Conclusiones}

Las construcciones de posesión externa en mazahua se codifican a través de un sufijo de dativo; estas construcciones se comportan sintácticamente como las oraciones de objeto indirecto. La entidad poseída se encuentra altamente afectada, la intención comunicativa del hablante es la de poner en foco o la de resaltar al poseedor. 
Los rasgos bajo los cuales se presenta la construcción de posesión externa son los siguientes: la frase nominal que expresa la relación poseído-poseedor lleva una marca de posesión y esta frase aparece básicamente en posición postverbal. En posición preverbal es posible que aparezca la frase poseída si ocurre en primera posición la frase nominal que expresa a la entidad poseída y luego aparece la frase nominal que expresa a la entidad poseedora. El prefijo que codifica al poseedor se asimila al sufijo de dativo.

La entidad poseída es semánticamente muy amplia, esto es, relaciones de parte todo, relaciones de parentesco y posesiones en general, como comida, ropa, dinero, animales, vivienda, instrumentos laborales, musicales y de cultivos. El poseedor es una entidad animada y puede aparecer expresado en primera, segunda y tercera persona. Los estados de cosas expresados en las construcciones básicamente codifican actividades, achievements, acomplishments que en lo regular pueden o no afectar al paciente. La entidad poseída sólo se expresa en el objeto directo.

Finalmente, la posesión externa no se codifica con locativos y con verbos de estado. El evento no debe ser reflexivo y no debe haber correferencia entre el sujeto y el poseedor.

\section{Referencias}

Aikhenvald Alexandra Y. y R.M.W. Dixon 2013. Possession and ownership. A crossLinguistic Typology. Oxford: Oxford University Press.

Arellanes, Francisco; Alejandra Ortíz; Armando Mora y Antonio García 2014. "Syntax of the non-eventive semantic relations in four Otomanguean languages: Amuzgo, Mazahua, Mazatec and Zapotec". $6^{\text {th }}$ International Conference Syntax of the World's Languages: Pavia (Italia).

Beavers, John 2011. "On Affectedness". Natural Language \& Linguistic Theory 29. 335-370.

Dowty, David 1991. "Thematic proto-roles and argument selection". Language 67:547619.

Fidencio, Verónica 2012. "Alomorfía del objeto directo e indirecto en Mazahua de San Pedro del Rosal". V Coloquio de Lenguas Otomangues y Vecinas. México: Fray Antonio de los Reyes. Oaxaca.

Fried, Mirjam 1999. "From Interest to Ownership A Constructional View of External Possessors", en External possession. Payne, Doris L. y Immanuel Barshi (eds). Amsterdam: John Benjamins Publishing, pp 473-504.

Knapp, Michael 2008. Fonología segmental y léxica del mazahua. México: INAH.

Haspelmath, Martin 1999. "External Possession in a European Areal Perspective", en External possession. Doris L. Payne y Immanuel Barshi (eds). Amsterdam: John Benjamins Publishing, pp 109-136. 
Hopper, Paul J. and Sandra A. Thompson 1980. "Transitivity in grammar and discourse". Language 56:251-299.

Mithun, Marianne. 1984. "The evolution of Noun Incorporation", en Languages 60: 847-894.

Mora-Bustos, Armando 2012. "Relaciones gramaticales en mazahua". Seminario de complejidad sintáctica. México: Universidad de Sonora.

Mora-Bustos, Armando 2015. "Los verbos ditransitivos en mazahua”. Liames 15: 83103.

Palancar Enrique y Roberto Zavala (eds) 2013. Clases léxicas, posesión y cláusulas complejas en lenguas de Mesoamérica. México: Publicaciones dela casa Chata.

Palancar Enrique 2008. "Posesión externa en otomí". Estudios de cultura otopame. 6: 263-279.

Palancar Enrique 2009. Gramática y textos del hñoñho. Otomí de San Ildefonso. Tultepec, Querétaro. México: Universidad de Querétaro y Plaza y Valdes.

Payne, Doris L. y Immanuel Barshi (eds) 1999. External possession. Amsterdam: John Benjamins Publishing.

Peralta Ramírez, Valentín 2013. "Posesión externa en el náhuatl de Amanalco", En Clases léxicas, posesión y cláusulas complejas en lenguas de Mesoamérica. Palancar Enrique y Roberto Zavala (eds). México: Publicaciones dela casa Chata. pp. 103-131.

Quintana Toribio, Elizabeth 2012. Marcación de la posesión en la lengua mazahua de San Juan de las Manzanas, Edo. Mex. (2010-2012). México: Universidad Intercultural del Estado de México. Tesis de licenciatura.

Stewart, Donald 1966. Gramática del mazahua. Correcciones y comentarios de Doris Bartholomew. ILV: México. (Manuscrito).

Vargas Bernal, Gloria 2013. Alineamiento e intransitividad escindida en mazahua. México. CIESAS. Tesis de maestría.

Data recebimento: $20 / 01 / 2015$

Data aceite: $10 / 04 / 2015$ 